\title{
Research of Combined Combat Operational Plan Assessment Based on Dynamic Evolvement
}

\author{
Feng Wang, Zhinian Gao, Shoulin Shen, Changping Yuan \\ Army Command College \\ Nanjing, 210045, China. \\ wf19821007@yahoo.com.cn
}

\begin{abstract}
The most direct approach of evaluating the operation plan is to make use of the combat results which were obtained by evolving along the time axis according to the process of operation plan contents. Based on the understanding, the method of the combined combat operational plan based on dynamic evolvement is studied through combining utility model with dynamic evolvement platform based on MultiAgent in this paper. First, according to the characteristics of operational plan for combined-arms combat, the operational plan evaluation model based on the utility is established in the cognitive rule of utility. Second, according to a scenario of combined combat, four specific operational plans were developed. On these bases, dynamic evolvement experiments for these operational plans were implemented on the MultiAgent experimental platform, and the data from these experiments was used for evaluating which operational plan is the best. Finally, the advantages and disadvantages were pointed out in the research.
\end{abstract}

Keywords-Operational plan assessment, Utility function, Dynamic evolvement, Cognitive rule

\section{INTRODUCTION}

Combined-arms combat is the basic operational form of Army' division, brigade and regiment forces in local wars involving high-technology. Currently, the nations militaries are positively transforming from the mechanization into the informatization by the vigorous promotion of the new military revolution. Under the influence of the revolution, the complexity of combined combat is emerging gradually, and bringing many new great challenges to program and assess the combined combat operational plan. What is the operational plan of satisfying with objective reality? Is there the optimal operational plan? To answer these questions, the most direct method is to evolve the operational plan along the time-axis according to the contents and procedures of the operational plan until the combat finishes, and make use of the combat result to assess the operational plan. Based on this understanding, the method of combined combat operational plan assessment based on the dynamic evolvement is studied in this paper.

\section{ASSESSMENT OF THE OPERATIONAL PLAN UTILITY}

\section{A. Cognitive Rule of the Operational Plan}

The utility of the operational plan is the form of the commander's concept on the operational plan attributes. According to the points of cognitive psychologists
Montgomery, the commander would make use of the following six criteria to choose the operation plans ${ }^{[4]}$ :

1) Dominance rule: The plan has at least one attribute better than the other plans', at the same time, and the other attributes no better than the other plans'. Obviously, when the rule is applicable for assessment, the commanders would get the same conclusion even though the commanders have the different subjective preferences. But, it must be noted that the rule can only be used in very limited circumstances.

2) Conjunctive rule: each attribute of the plan is not inferior to a certain threshold, and this pattern is applicable to the optimization process of the plan.

3) Disjunctive rule: The plan' attributes which should not be below the given threshold are more than one.

4) Lexicographic rule: The attributes of the plan are ordered based on the importance of the attributes, and the assessment is to make the most important attribute of the plan as the criterion to choose the strategy plan.

5) Attractive rule: An attribute of the plan has the incomparable superiority to the other plans', and can be used to choose the plan directly.

6) Additional rule: The pairwise comparisons of the plan' attributes are used to confirm the weight of each attribute, and then the strategy plan would be chosen according to the weighted value of each attribute.

\section{B. Utility Function of the Operational Plan}

The set of operational plans which wait for being evaluated is $P=\left\{p_{l}, p_{2}, \cdots, p_{m}\right\}$. The attribute set of the results oriented from the operational plan evolvement is $X=\left\{x_{1}, x_{2}\right.$, $\left.\cdots, x_{n}\right\}$. Reasonably choosing the elements from the set of operational result attributes $X$ is significant for the assessment of the plan.

Matrix $C$ is defined by $h: P \times X \rightarrow C . h$ expresses the evolvement process of the operational plan, and $C$ is the matrix which is made up of the attribute values of the combat result. $U=\left\{U_{1}, U_{2}, \cdots, U_{n}\right\}$ expresses the set of the utility function which is made up of the attributes, $U$ is the commanders' relative preference on the attribute value $C$. According to the reference[2][3], if and if only $U_{j}\left(C_{i j}\right) \geq U_{l}\left(C_{k l}\right)$, the commander thinks that the result $C_{i j}$ is better than the results $C_{k l}$. The attribute value $C_{i j}$ can be transformed into the utility value $U_{i j}$ with the utility function. The transformation relationship can be expressed as follows: 


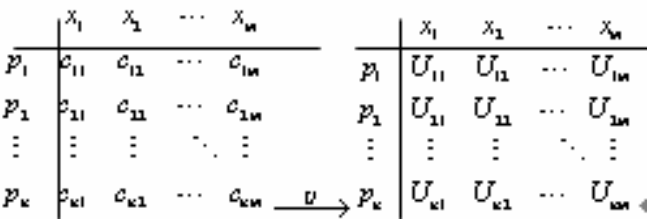

Under the five cognitive rules, the utility function of the operational plan could be uniformly expressed as follows:

$$
P\left(p_{j}\right)=\sum_{j=1}^{n} U_{j}\left(c_{i j}\right)
$$

Thereinto,

Thereinto,

$$
U_{j}\left(c_{i j}\right)= \begin{cases}k & \text { if better than the disjunction rule threshold } \\ -K & \text { if less than the disjunction rule threshold } \\ g_{j}\left(c_{i j}\right) & \text { others }\end{cases}
$$

$$
k>\max _{i}\left\{\sum_{j=1}^{n} g_{j}\left(c_{i j}\right)\right\}, \quad K=2|k|+\varepsilon
$$

$\varepsilon$ is the any positive number. To complete the construction of the utility function, it is necessary to structure the function $g_{j}$. Structuring the function $g_{j}$ should satisfy with three basic conditions: (1)The function $g_{j}$ should be able to scale property value $c_{i j}$, and make these values be compared with each other. (2)The function $g_{j}$ should be able to judge whether the attribute value is to increase the utility of the operational plan or reduce the utility of the operational plan. (3)The function $g_{j}$ should be able to incarnate the operational plan result's attribute weight given by the commander. Hereto, the construction of the operational plan utility function is completed.

\section{Preparation for the EVOlVEMEnT OF the OpERATIONAL PLAN}

\section{A. Confirming The Assessment Index}

Assessment indexes are the key of transforming the operational plan from the "perceptual concepts" to "rational measurement", but, with regard to the different combats, the assessment indexes of the dynamic evolvement are very different due to the different concerns of the commanders and the command organs of different concerns. So, the assessment indexes are not static and immutable, and need to be confirmed dynamically based on the requirements of the commanders and the operations. For example, in one offensive combat of the mechanized infantry regiment, the key problems which are concerned by the commander and the command organ include the following: the first is the form of the force disposition; the second is the main operational direction; the third is the force wastage and ammunition decrement.

The mechanized infantry regiment's operational task given by the senior is to occupy the enemy's defensive position, while making a request for the loss of our main battle weapons. Based on the combat tasks and the problems concerned by the commander, the following five attributes of the combat results are chosen to be the assessment indexes of the operational plan of the mechanized infantry regiment offensive combat
(1) Whether occupying the enemy position or not $\left(x_{1}\right)$. (2) Wastage of our main battle weapon $\left(x_{2}\right)$. (3) Combat duration $\left(x_{3}\right)$. (4)Wastage of the enemy's main battle weapon $\left(x_{4}\right)$. (5) Our consumption of various types of ammunition $\left(x_{5}\right)$.

\section{B. Configuration Of The Operational Plan'S Experiment}

1) Experimental platform:

The evolvement platform based on Multi-Agent made use of JAVA as the development language, and the nonpreemptive threading technology of the Java VM as the core simulation engine. The platform is that: (1) it was developed by using the modeling method based on Multi-Agents; (2) it had ample ability aggregation; (3) Agent can be endowed with multi-capabilities, including the shoot capability, the reconnaissance capability, the command capability, the communication capability and so on.

2)Map: the specific map of $f$ area

Scale: 1:50000

The battlefield was divided into $100 * 100$ grids after the quantification process. The elevation of each grid was calculated according to the swatch average elevation of the grid. The generated file was called $f$.trn, and stored in the database.

\section{3) Time constraints:}

The minute was the unit of the simulation time. 1 second which is the simulation time was equal to 1 minute which is the standard combat time. When assessing the plans, the combat time could be compared according to the simulation time.

\section{4) Input the evolvement plan}

According the offensive combat scenario of the mechanized infantry regiment, we made use of the different combination the force disposition with the main operational direction to establish 4 operational plans $p_{1}, p_{2}, p_{3}$, and $p_{4}$, and then enter them into the evolvement platform one by one.

\section{EVOLVEMENT RESULT ASSESSMENT}

Each plan was run four times. One of evolvement process of the first plan $p_{l}$ was shown in Figure 1.

The essential of judging the utility of the operational plan is to structure $g_{j}$. According to the three basic conditions which the function $g_{j}$. need to satisfy, the function $g_{j}$ could be structured based on the following steps:

Step 1: Confirm the scale factor of the attribute $v_{j}$. It is mainly to solve the problem of inconsistent measurement unit of each attribute. According to the combat task of the mechanized infantry regiment, we could know that: the attribute $x_{l}$ has the right of "one-vote veto"; its disjunction threshold is $x_{1}=1$; its scale factor could be any positive real number. According to the attribute matrix, it can be seen that the attributes $x_{2}, x_{3}, x_{4}$ and $x_{5}$ had little difference, and could be respectively set to $-1,-1,-1$ and +1 as the scale factor of each other after ignoring the impact of units of measurement.

Step 2: Confirm the attribute significance factor $w_{j}$. The significance factor is confirmed by the preference of the commander. Through the experiments, the psychologists 
G.A.Mille indicated that the grads which can be correctly distinguished by everyman are from 5 grads to 9 grads, and ought to try the best to use 9 grads in order to improve the accuracy if possible. Based on multi-attribute utility function, the utility of each operational plan could be estimated according to the attribute matrix of the plan evolvement result, as shown in Table 2According to Table 2, the sequence of operational plans' utility is that $p_{3} \succ p_{1} \succ p_{4} \succ p_{2}$ 。

The results showed that the third operational plan is the best in all plans.

It should be noted that: although the result of the assessment was that the $3^{\text {rd }}$ operational plan was the optimal, but it didn't mean that the $3^{\text {rd }}$ operational plan was perfect. When optimizing the plan, we could track the specific changes of the plan along the simulation evolvement trajectory oriented from the experiment, and find the bugs of them, which made the optimization have a definite object in view.

The offensive combat of mechanized infantry regiment is a typical pattern of the combat, and has the representativeness. With regard to the other combat styles, the same methods and steps can be still used to assess the operational plans after confirming the appropriate assessment contents and indexes.

\section{SUMMARIZE}

Although the traditional methods of assessing the operational plan, such as the GAHP method, the neural network method, the fuzzy comprehensive evaluation method, the Survivability method and so on, can also meet the requirement for assessing the operational plan in a certain extent, but all of them are the results the comprehensive illation, and difficult to assess the impact of the chanciness and the emergence on the operational $\operatorname{plan}^{[7][8][9]}$. In addition, the purpose of the operational plan assessment is not only to select the optimal, but also optimize the operational plan. But the traditional assessment methods lack the ability of explaining the operational plan, thus it is difficult for them to play roles in deeper application. In contrast, oriented from the bottom level cognition of the combat, the method discussed in the paper can not only assess the established operational plan, but also effectively make use of the advantage of the simulation platform based on Muti-Agent to observe, explain and analyze the high level behaviors showed in the process of implementing the operational plan. According to these, we can identify the deficiencies of the plans, and make the optimization of the plan have a definite object in view.

Of course, many details of the method still need to be further improved in future. For example, the diversity of the assessment model need to be further enriched, the assessment indexes need to be further optimized and refined. In addition, the objectivity of the assessment is strongly dependent on the data obtained from the "experimental experiences", and thus, the simulation fidelity of the dynamic evolvement platform based on Muti-Agent need to be further improved.

\section{REFERENCE}

[1] GUO Anhua, ZENG Sunan. History of the Development of Combined Combat[M]. Beijing: Liberation Army Press,2008.01.

[2] Bernoulli D. Exposition of a new theory on the measurement of risk. Econometrica, 1954,22(1):23-36

[3] Fishburn PC. Independence in utility theory with whole product sets. Operations Rearsh,1965,13(1): 28-45

[4] Henry Montgomery. Decision Making Cognitive Psychology, chapter 6,pages 171-188.Studentlitteratur, 1992

[5] ZHANG Zuilang. Military Operations Research[M]. Beijing: Military Science Academy,2005.09.

[6] Andrew IIachinski. Artificial War: MultiAgent-Based Simulation of Combat[M]. Beijing: Publishing House of Electronics Industry,2010.08. Survivability[J]. Fire Control \& Command Control, 2003.06,28(3):56 59.

[7] YANG Weilong, JI Jinyao, TANG Chuncan. Application of Fuzzy Comprehensive Evaluation Method Based on GAHP on Submarine Combat Scheme Evaluation[J]. Command Control \& Simulation, 2009.08,31(4)68 72.

[8] WANG Jingyan, ZHANG Jianjun, WU Yushu. A Method for Operation Plan Assessment Based on Neural Network[J]. Military Operations Research and Systems Engineering, 2005.03,19(1):57 61.

[9] YANG Ping, BI Yiming, LIU Weidong, etc al. The Research of Simulation Model for Operational Plan Evaluation Based on

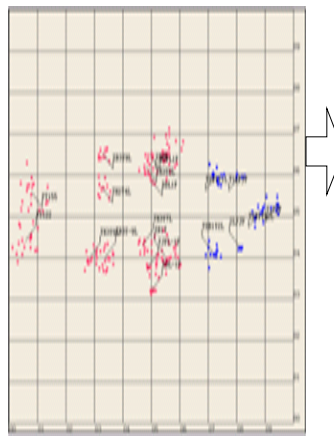

$1=0$

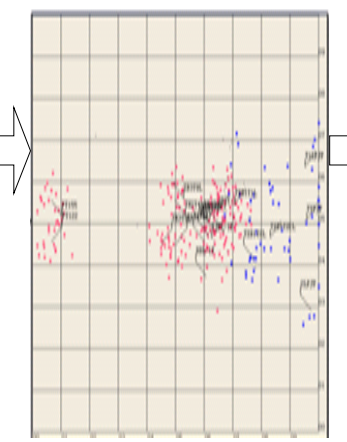

$E=30$

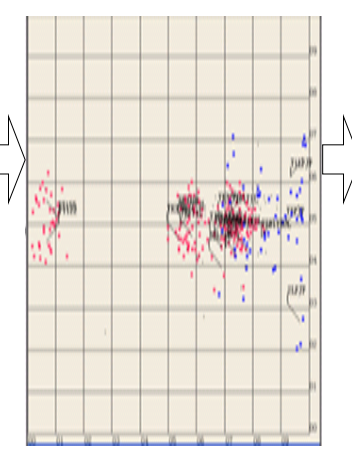

t=60

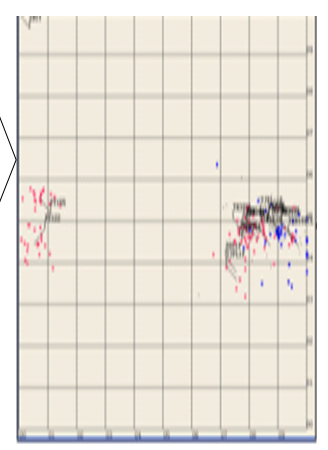

End

Figure 1. the process of the first operation plan $p_{1}$ evolvement 
TABLE I. SIGNIFICANCE FACTOR OF ATTRIBUTES

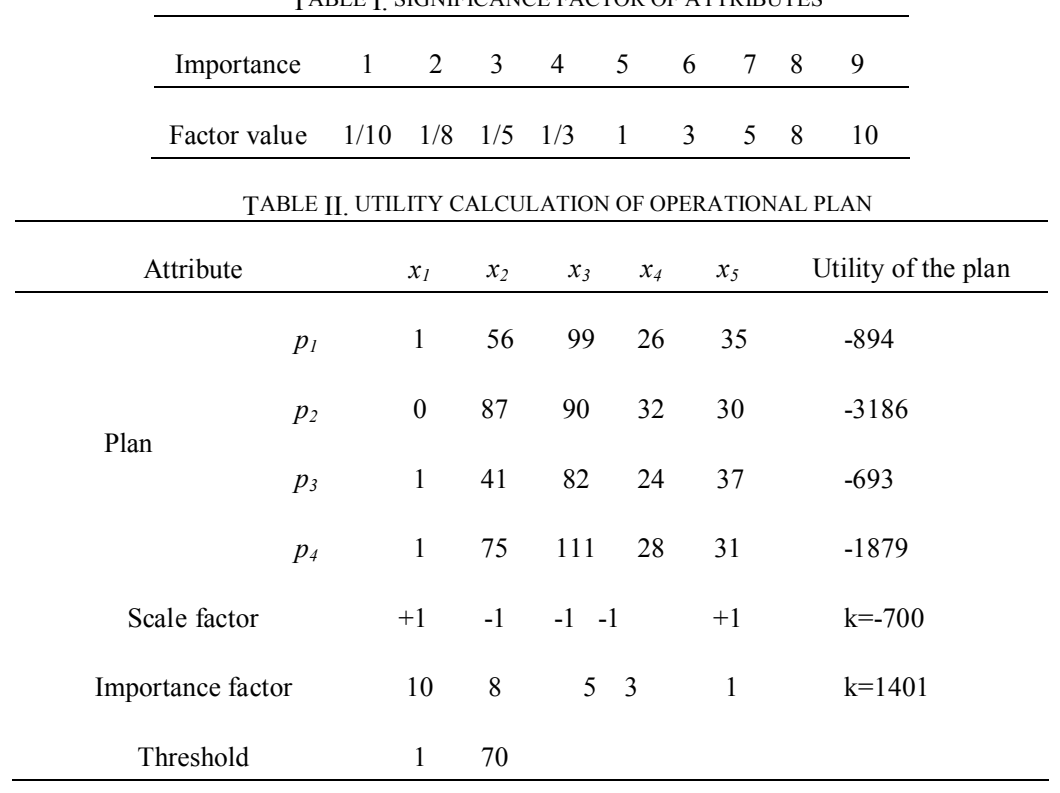

